\title{
Subcellular Level Resolution Fluorescence Measurements in Yeast Cells by Image Analysis
}

\author{
Evgeny Puchkov
}

\begin{abstract}
Computer image analysis algorithms of the ImageJ software (National Institutes of Health, USA; http://rsb.info.nih.gov/ij) have been tailored for subcellular microfluorimetry of the single Saccharomyces cerevisiae yeast cells. The algorithms were used for the quantitative analysis of digital images acquired by wide-field fluorescence microscopy combined with color digital photography aiming to investigate the intracellular distribution of anthracycline anticancer drug doxorubicin and to evaluate viscosity in the vacuoles of $t$ he cells.
\end{abstract}

Keywords - computer image analysis, fluorimetry, fluorescence anisotropy, ImageJ, yeast cells, Saccharomyces cerevisiae, fluorescence microscopy, viscosity, vacuoles, anticancer drug, doxorubicin

\section{Introduction}

Fluorescence measurements at subcellular level (microfluorimetry) of living cells are in demand for studies in many areas of life sciences. The most important advantage of this research tool lies in its ability to serve as a means for measuring spatial distribution of fluorescing molecules and, finally, structure-function relationships inside the cells (Bolte, \& Cordelieres, 2006; Ronneberger, et al. 2008; Tsygankov, et al. 2014). Currently, microfluorimetry can be performed by two technologies. The first can be executed by using the fluorescence microscopes of new generation (Combs, 2010). These microscopes enable microfluorometry by direct photometry through the use of high sensitive photomultipliers or avalanche photodiodes as detectors and lasers for fluorescence excitation. The second, or indirect technology, is based on the fact that color digital images of fluorescing objects acquired by a CCD or CMOS camera hold information on their fluorescence intensity, spectra, and 2D positions (Waters, 2009). Thus, indirect microfluorometry can be performed by computer image analysis of the digital color fluorescence microscopy images obtained by comparatively simple conventional fluorescence microscopy combined with digital photography. However, to use this approach in specific cases, custom quantitative image analysis algorithms for image analysis software are needed.

The yeast Saccharomyces cerevisiae is an object of a large research interest for at least two reasons. First, it is widely used in food industry, including baking and production of alcoholic beverages, ethanol and food additives. Second, it serves as a model system for studies of eukaryotic cells since many of the basic cellular properties

Evgeny Puchkov

Skryabin Institute of Biochemistry and Physiology of Microorganisms Russia between yeast and humans are highly conserved. This is also due to the availability of the DNA sequence of the complete genome and biochemical data, convenience for molecular manipulations and the ease of handling (Botstein, et al., 1997). The yeast cells of $S$. cerevisiae are shown to be a good model in many areas of cancer research (Nitiss \& Heitman, 2007) and for new drug discovery (Mager \& Winderickx, 2005). Hence, the development of new techniques and approaches for the studies of the yeast $S$. cerevisiae is an important task.

In this study, three computer image analysis algorithms of ImageJ software (National Institutes of Health, USA; http://rsb.info.nih.gov/ij) for microfluorimetry were designed to perform investigations on live (not fixed) $S$. cerevisiae yeast cells with the following objectives. 1. To test if intact yeast cells can be used as a model for locating intracellular sites/targets of the DNA-directed drugs with anthracycline anticancer drug doxorubicin as an example. 2 . To characterize quantitatively the movement of insoluble polyophosphate complexes (IPC) in the vacuoles of the cells and to evaluate the apparent viscosity in the vacuoles using the obtained data. 3. To evaluate the apparent viscosity within the vacuoles of the cells by steady-state fluorescence anisotropy measurements of quinacrine using wide-field fluorescence polarization microscopy.

\section{Materials and Methods}

\section{A. Preparation of Specimens for Microscopy}

A type strain of $S$. cerevisiae VKM Y-2549 was used in most of the studies presented. The culture was maintained on slants of agarized $(1.5 \%)$ wort $(4 \%)$ medium at $4{ }^{\circ} \mathrm{C}$. The cells from the slants were seeded into $20 \mathrm{ml}$ of a liquid medium containing $0.63 \%$ yeast nitrogen base (Becton Dickinson Microbiology Systems, USA) and $2 \%$ glucose in conic flasks. The culture was incubated at $25 \pm 0.5{ }^{\circ} \mathrm{C}$ for 18-24 h. Cells were concentrated by centrifugation up to approx. $5 \times 10^{8}$ cells $/ \mathrm{ml}$ and samples were supplemented with fluorochromes. The following final concentrations of the fluorochromes were used: 4,6-diamidino-2-phenylindole, dilactate (DAPI) (Sigma, USA) - $3 \mu \mathrm{M}$; ethidium bromide (E) (3,8-diamino-5-ethyl-6-phenylphenanthridinium bromide) (Serva, Germany) - $10 \mu \mathrm{M}$ and doxorubicin (DR) (Ferane-Bryncalov, Russia) - $10 \mu \mathrm{M}$; quinacrine $-200 \mu \mathrm{M}$. After incubating at $25 \pm 0.5{ }^{\circ} \mathrm{C}$ for appropriate time (depending on the experiment), $6 \mu \mathrm{l}$ aliquots of the cell suspension were placed onto specimen slides, covered with cover glass, and sealed with nail polish on the periphery of the cover glass to prevent evaporation. Observations were started ca. 20 min after sealing, when most cells had stuck to the slide's glass. Only obviously still cells were photographed. 


\section{B. Wide-Field Fluorescence Microscopy, Color Digital Photography and Conventional Fluorimetry}

Fluoresce microscopy and color digital photography were carried out on a ML-2B fluorescence microscope (LOMO, Russia) equipped with a Sony DSC-V3 digital camera. By applying appropriate filter sets, the following combinations of $\lambda_{\mathrm{ex}} / \lambda_{\mathrm{em}}$ were used: for DAPI, DR and E $360 \mathrm{~nm} />400 \mathrm{~nm}$; for DR and E - $430 \mathrm{~nm} />520 \mathrm{~nm}$; for quinacrine $-400 \mathrm{~nm} />510 \mathrm{~nm}$. In the light transmission mode of operation, a combination of filters with transmittance $\lambda_{\max }$ near $520 \mathrm{~nm}$ in front of an object and a ZhS-3 filter (transmittance $\lambda>400 \mathrm{~nm}$ ) behind it were installed. For the quinacrine polarization studies, in the fluorescence excitation and emission light paths, polarizers from the polarization accessories of a Hitachi 850 spectrofluorimeter (Hitachi, Japan) were installed. Images were acquired in two camera modes. The first was the default mode of photographing with automatic selection of sensitivity. In the IPC Brownian motion studies, the speed regime (Speed Burst option of a Sony DSC-V3 digital camera) was used. It gave series of eight frames with an interval of $0.43 \mathrm{~s}$ and an interval between series $2-3 \mathrm{~s}$. For the publication, the color images were transformed into the 8-bit gray ones. Conventional fluorimetric measurements of the dyes in solutions were conducted on a Hitachi 850 spectrofluorimeter (Hitachi, Japan). In case of anisotropy measurements of quinacrine in the sucrose solutions, the polarization accessories provided with the instrument were used.

\section{Software}

The color digital images of the fluorescing cells were processed by the Adobe Photoshop, v. 8.0 (Adobe Systems Inc., USA) and analyzed by the ImageJ 1.42 (National Institute of Health, USA, http://rsb.info.nih.gov/ij) software. Quantitative treatment of the data was made with MS Excel 2003 and OriginPro 7.5 (OriginLab, USA) software.

\section{Results and Discussion}

\section{A. Intracellular Distribution of Doxorubicin}

For better understanding of both therapeutic and side/toxic effects of drugs, their intracellular location/distribution needs to be elucidated. Because many, if not all, DNA-targeted drugs are fluorophores, this can be done by fluorescence microscopy with mammalian cell lines as an in vitro model (Egorin, et al., 1980; Giglia, et al., 1988). However, this model is expensive, tiresome and restricted in potential molecular or genetic manipulations.

The aim of this study was to test if intact (not fixed) yeast cells of $S$. cerevisiae can be used as an inexpensive and easy to handle model for locating intracellular sites/targets of the DNA-targeted drugs.
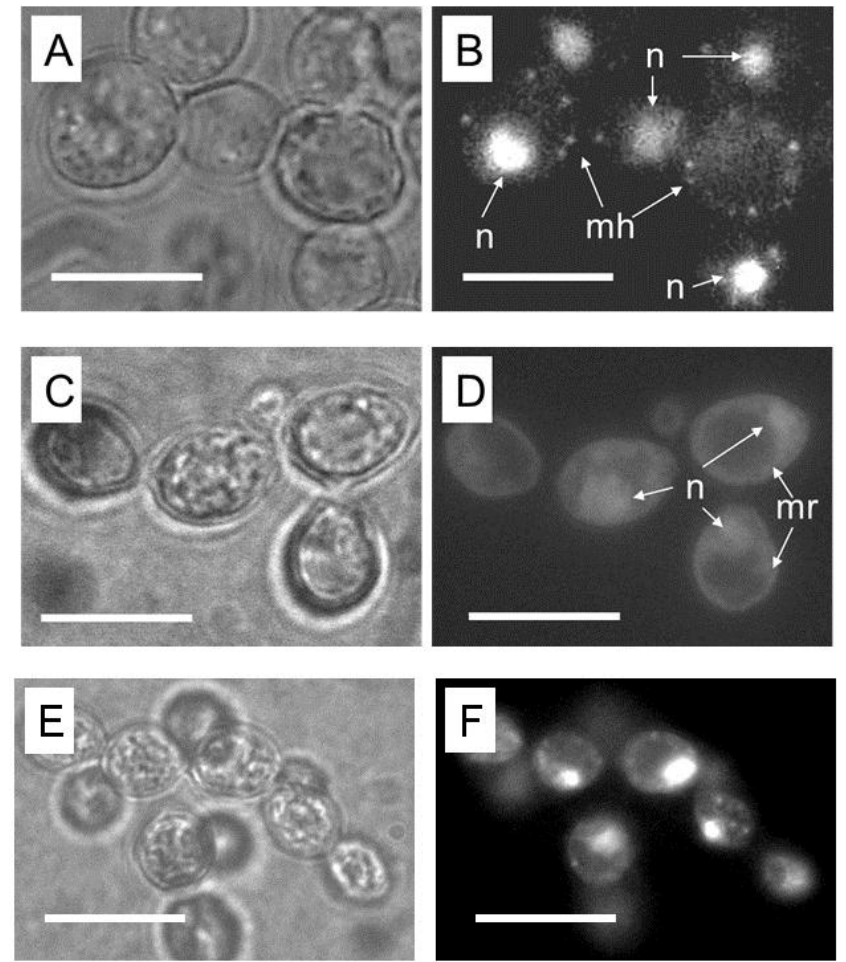

Figure 1. $S$. cerevisiae yeast cell microscopic images: A, C, E transmission mode; B, D, F - fluorescence mode. A, B - DAPI-stained cells; C, D - DR-stained cells; E, F

(DR+DAPI)-stained cells. $\mathrm{n}$, nuclei; mh, mitochondria; mr, mitochondrial region. $\mathrm{Bar}=10 \mu \mathrm{m}$.

To this end, intracellular distribution of anthracycline anticancer drug doxorubicin (DR) (trade name Adriamycin), which is known to interact with nucleic acids (Yanga, et al., 2014, and reference therein), was investigated along with fluorescent nucleic acid dyes ethidium (E) (Le Pecq, \& Paoletti, 1967) and DAPI (Suzuki, et al., 1997) as intracellular DNA markers, using fluorescence microscopy combined with computer image analysis.

In the cells with DNA stained by DAPI, there were clearly visible fluorescing blue spots of nuclei and small dots of mitochondria (Fig. 1, B) (Williamson \& Fennell, 1979). As compared to DAPI, intracellular distribution of yellow-orange DR fluorescence (Fig. 1, D) and that of orange-red E fluorescence (not shown) had not the clear "spot and dot" appearance. Application of DAPI in combination with DR or E visualized location of the nuclei and the mitochondria by "spot and dot" appearance similar to that of DAPI staining alone (Fig. 1, F).

To interpret the visual observations and more fully characterize intracellular DR binding structures, particularly to answer the question whether DR was located in the sites of the DAPI stainable DNA, computer image analysis was performed. With this goal in mind, the following algorithm of ImageJ was designed. Red, green and blue components of the fluorescence intensity were quantitatively assessed in the selected subcellular regions of interest (ROI "Oval Selection") of the cell images using "Analyze" plugin ("Measure RGB" option). The obtained data (Fig. 2, insets 2 - 4) were called 'pseudospectra' as they roughly corresponded to the real spectra of the dyes (Fig. 2, inset 1). 

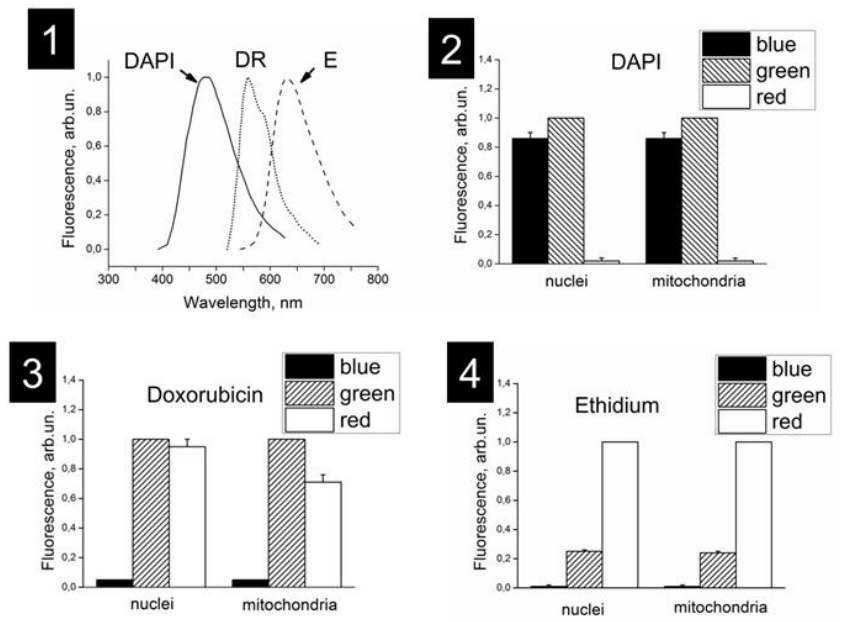

Figure 2. Single yeast $S$. cerevisiae cell fluorescence pseudospectral analysis. 1 - normalized fluorescence spectra of DAPI, DR and E in water. 2, 3, 4 - fluorescence intensities of DAPI, DR and E in the nuclei and in the mitochondria in blue, green and red regions (pseudospectra) measured after RGB-split of the fluorescence images.

Using this approach, called 'pseudospectral analysis', it was shown that all three dyes were located in the nuclei and in the mitochondria. The red component of the DR pseudospectrum in the nuclei was more pronounced than in the mitochondria (Fig. 2, inset 3). These data indicated that, for DR molecules bound in the nuclei, the fluorescence spectrum has a more pronounced red part than for molecules bound in mitochondria. DR fluorescence spectrum has a red region "shoulder". Upon interaction with DNA, this part of the DR spectrum is known to increase (Angeloni, et al. 1982), while the shape of the DAPI (Haugland, 1996) and E (Le Pecq \& Paoletti, 1967) spectra do not change. Therefore, it was supposed that the red component of the DR fluorescence in the mitochondrial region is less pronounced because a fraction of the molecules was bond to some other than DNA structures. Most probably these structures were the mitochondrial membranes. Potential ability of DR binding to biological membranes was demonstrated on artificial membranes by others (Gallois, et al. 1996). Membrane binding may also explain the diffuse fluorescence of DR in the mitochondrial region as distinct from the dot-like pattern of the mitochondrial DNA staining by DAPI (Fig. 1, D). The distribution of $\mathrm{E}$ fluorescence in the mitochondrial region was similar to that of DR (not shown). As E was demonstrated to being able to incorporate into the isolated mitochondrial membranes (Le Pecq \& Paoletti, 1967), it can be suggested that this nucleic acid dye was bond in the mitochondria to both DNA and the membranes.

An essential distinction of the pseudospectrum of DAPI from pseudospectra of DR and E is the lack of the red component and the presence of the pronounced blue component (Fig. 2, insets $2-4$ ). This distinction was used for evaluating codistribution and colocalization (Dunn, Kamocka, \& McDonald, 2011) of DR and E with DAPI in the cells by analyzing pseudospectra upon combined addition of DAPI with DR or E. It was found that, in the DAPI locations, both in the nuclei and in the mitochondria the red component was greater as compared with DAPI alone, though smaller than with DR alone. If the cells were first incubated with DR and then DAPI was added, the blue component almost did not change, and the red component was somewhat higher than upon simultaneous addition of the dyes. In case of DAPI + E combination, the intensity of the red component did not depend on the order of addition.

Appearance of the red fluorescence component in the regions of DAPI stained DNA upon combined application of DAPI with DR and $E$ is an indication of colocalization of DAPI with DR and E in the nuclei and in the mitochondria. In the sites of DR and E colocalization with DAPI, the red component of their fluorescence was less as compared to the application of DR and $\mathrm{E}$ alone. These data can be interpreted as a competition of DAPI with DR and E for the same DNA binding sites. No data in the literature on DAPI competition with DR and $\mathrm{E}$ for binding to isolated DNA were found.

\section{B. Characterization of Insoluble Polyphosphate Complexes' Movement}

In the $S$. cerevisiae cells, at some cultivation conditions, vividly moving particles, of $<1 \mu \mathrm{m}$ in size, known as 'dancing bodies', appear. They were shown to be insoluble polyphosphate complexes (IPC; Wiame, 1947) localized in the vacuoles (Urech et al., 1978).

It is assumed that the 'dances' of the IPCs is Brownian motion, although, strictly speaking, this has not been proved. If so, then measurements of the parameters of their movement could provide insight into the physical properties of the medium inside the vacuoles.

The Brownian motion of particles obeys the EinsteinSmoluchowski equation (Saks et al., 2008;Berg, 1983, and references therein), which for two-dimensional (2D) movement is as follows:

$$
\left\langle s^{2}\right\rangle=4 \kappa T t / 3 \pi \eta D
$$

where $\left\langle s^{2}\right\rangle$ is the average of the square of displacement, $\boldsymbol{\kappa}$ is the Boltzmann constant, $\boldsymbol{T}$ is the thermodynamic temperature, $\boldsymbol{t}$ is the elapsed time, $\boldsymbol{\eta}$ is the viscosity and $\boldsymbol{D}$ is the diameter of the particle.

Thus, characterization of the IPC motion, as a kind of natural probe, may allow evaluation of apparent viscosity inside the vacuoles if their size and the average displacements can be measured. Insoluble polyphosphate complexes (IPC) can be revealed by DAPI staining in the vacuoles of some yeast cells as vividly moving yellow particles amid motionless blue nuclei and mitochondria (Fig. 3 , B). To characterize their movement, the following image processing algorithm of the ImageJ software was designed. Images of each eight frame Speed Burst set (see Materials and Methods) were corrected for Brightness/Contrast up to the same level, and appropriate magnification was chosen to make the fluorescing particles reliably visible. Then the images were transformed into 8-bit gray type. In a frame of a series, a fluorescing particle was selected as a region of interest (ROI) by Oval Selection option. ROI Manager option was switched on to get the same ROI area in other frames, although position of the ROI was changed according 

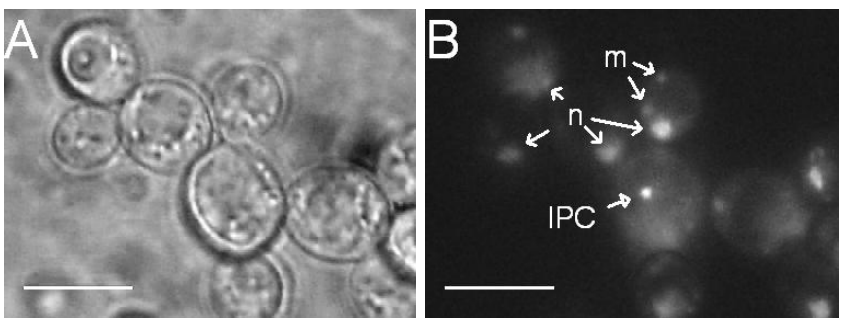

Figure 3: Insoluble polyphosphate complex (IPC) in the $S$. cerevisiae cells stained by DAPI. A. Transmission mode. B. Fluorescence mode. n, nuclei; $\mathrm{m}$, mitochondria (both fluoresce blue); IPC, insoluble polyphosphate complex (fluoresce yellow). Bars $=10 \mu \mathrm{m}$.

to the position of the particle in a new frame. Fluorescence intensity of the particles was assessed in $0-256$ units of the gray scale (option Mean Gray Value). For each eight frames' set, fluorescence intensities were normalized. The locations of particles in the two dimensional space (X and $\mathrm{Y}$ locations) were evaluated using Center of Mass option (Fig. 4 and 5). Fluorescence intensity profiles of particles were estimated by Plot Profile option after making Line Selection across the "center of mass".

As IPCs move in three dimensions, a criterion for selecting the 2D displacements in the photorecords, which are in the focusing plane or at least close to it, must be found. To learn how this could be done, experiments were performed on suspensions of fluorescein isothyocyanatelabeled latex microspheres of $2.1 \mu \mathrm{m}$ and $3.1 \mu \mathrm{m}$ diameter (Sigma, USA) in water $\left(2 \times 10^{5}\right.$ microspheres/ $\left.\mathrm{ml}\right)$. In this model, two parameters were known, the diameters of the microspheres and the viscosity of distilled water in which they were moving.

Using photorecords similar to those for IPC, two parameters of Brownian motion of fluorescing microspheres were estimated for each series of the eight speed regime shots - the consecutive two-dimensional locations and the mean fluorescence intensity served as a quantitative measure of the microsphere shift from the two-dimensional motion in each eight frames series of the speed regime shots. It was tested whether the fluorescence decrease of no more than $15 \%$ may be used as a criterion for taking displacements of the microspheres close enough to the two-dimensional space for viscosity assessment by formula (1). An example in Fig. 6 illustrates how this criterion was applied. In this example, only the displacements between points $1-6$ were measured and used for further calculations, while displacements between points $6-8$ were not, since fluorescence intensity in the point 7 decreased to more than $15 \%$ (Fig. 6, inset). Upon selecting "appropriate" displacements by this criterion from photorecords of the series of speed regime shots,
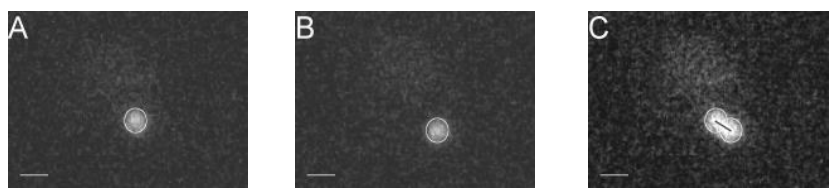

Figure 4. An example of the IPC location and displacement determination. $\mathrm{A}$ and $\mathrm{B}-$ two consequent frames captured with a $0.43 \mathrm{~s}$ interval. $\mathrm{C}-\mathrm{a}$ merge of A and B. White circles are selection areas (ROI) of the IPC. Black line connects the Centers of Mass of the IPC in two locations, it illustrates a displacement of the IPC for $0.43 \mathrm{~s}$. Bars $=1 \mu \mathrm{m}$.

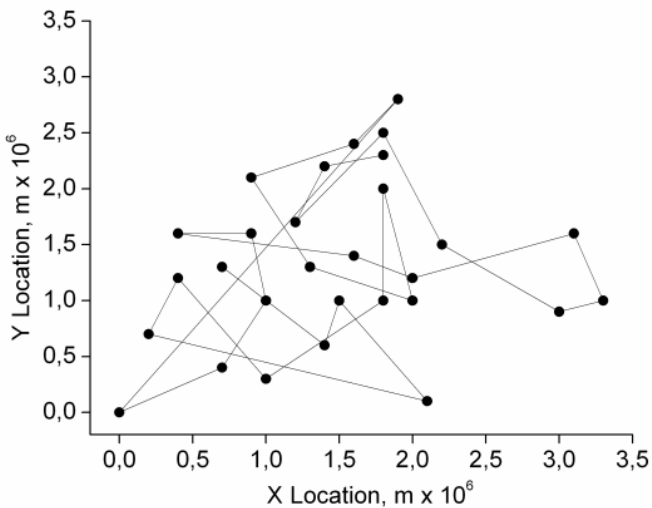

Figure 5. An example of consecutive $X$ and $Y$ locations of an IPC in a cell of $S$. cerevisiae. Ten series of the speed regime shootings in the fluorescence mode ( 80 frames, total) of the cell were analyzed. All the visible IPC locations are presented.

computation of the apparent water viscosity using formula (1) has been made. The viscosity values of water estimated by these measurements agreed with $30 \%$ accuracy with the value $\left(0,89 \mathrm{cP}\right.$ at $25{ }^{\circ} \mathrm{C}$, the temperature of measurements) obtained by other methods (Sheely, 1932). This result indicated that the developed approach may be used for viscosity assessment.

It was also found by analysis of the fluorescence intensity profile ("Line selection" across "Center of Mass" and "Plot Profile" options) of the microspheres that the level of the $85 \%$ fluorescence intensity corresponds to their outer borders (Fig. 7). That gave the means of estimating the size of the fluorescing IPC particles by their fluorescence intensity profiles. Using methodology developed on the latex microspheres, Brownian motions of the IPCs in four cells were analyzed. Displacements for each IPC in the twodimensional space in the series of the eight speed regime shots were estimated as it is shown in Fig. 8, for example. Outer borders of IPC were determined by estimating $85 \%$ level of fluorescence intensity in the IPC fluorescence profile. Fig. 9 illustrates how it has been done for one IPC. That gave the dimensions of IPC. Since the shape of IPC is not known, this parameter was assumed to be corresponding to the diameter of the microspheres.

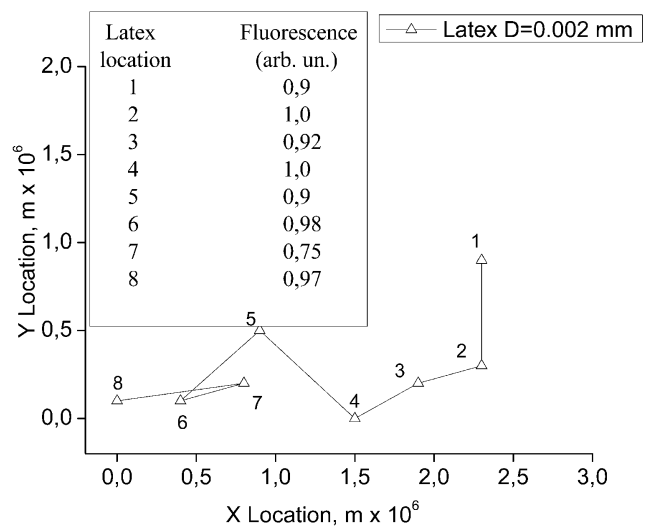

Figure 6. An example of analysis of a seven-step random walk of a fluorescing latex microsphere (diameter $=2.1 \mu \mathrm{m}$ ) using a series of the eight speed regime shots with a $0.43 \mathrm{~s}$ interval. Inset shows the microsphere fluorescence intensity at the corresponding locations indicated by numbers. 


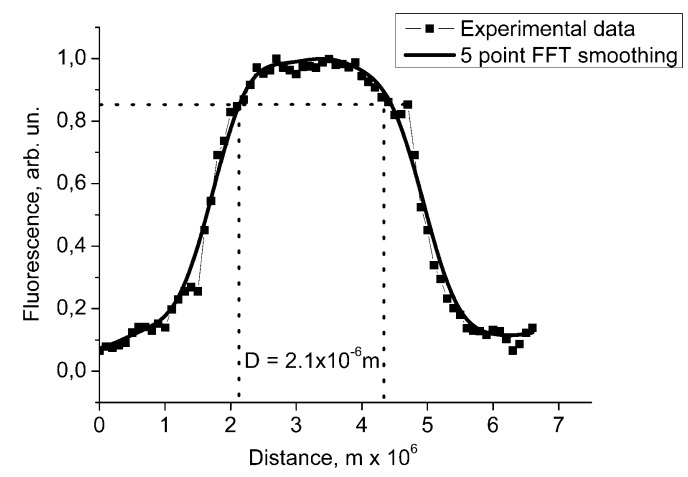

Figure 7. Fluorescence intensity profile of a fluorescing latex microsphere (diameter $=2.1 \mu \mathrm{m}$ ). Dotted lines show the level of the normalized fluorescence intensity $(0.85)$ corresponding to the diameter of the microsphere. (Similar results were obtained for the microspheres with $\mathrm{D}=$ $3.1 \mu \mathrm{m}$, not shown).

In four yeast cells, the 2D displacements and sizes of the IPCs were evaluated. Apparent viscosity values in the vacuoles of the cells computed by the EinsteinSmoluchowski equation using the obtained data, were found to be of $2.16 \pm 0.60 ; 2.52 \pm 0.63 ; 3.32 \pm 0.9 ; 11.3 \pm 1.7 \mathrm{cP}$.

\section{Quinacrine Fluorescence Anisotropy Measurements}

The approach presented above for the measurements of the apparent viscosity within the vacuoles has restrictions. First, it can be applied only for the cells with the vacuoles containing the IPC. Second, it is too laborious to be used for large number of cells to get information of the population level. For further studies of the apparent viscosity of the vacuolar milieu of the cells of $S$. cerevisiae, new methods are needed.

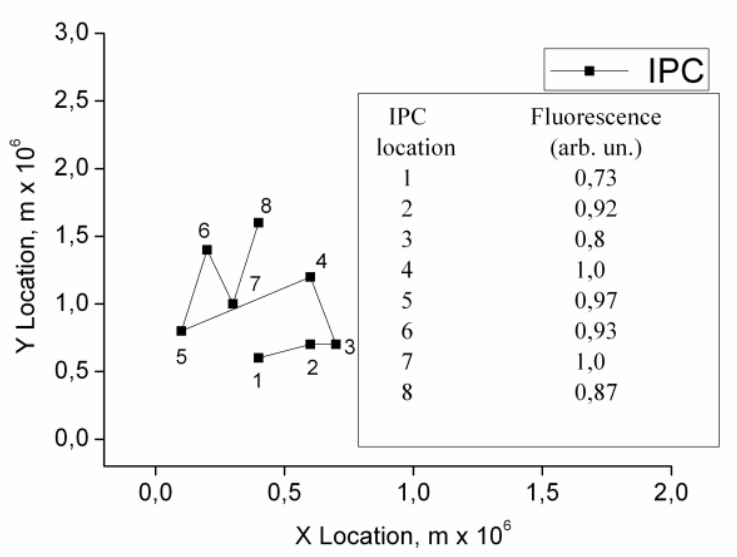

Figure 8. An example of analysis of a seven-step random walk of one IPC using a series of the eight speed regime shots with a $0.43 \mathrm{~s}$ interval. Inset shows the IPC fluorescence intensity at the corresponding locations indicated by numbers.

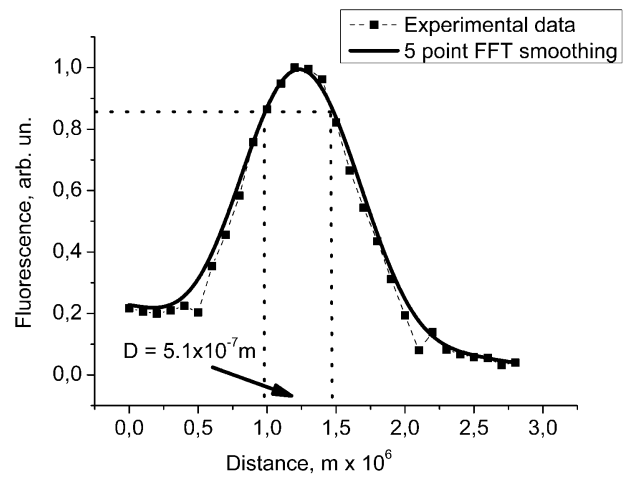

Figure 9. Fluorescence intensity profile of one IPC. Dotted lines show the level of the normalized fluorescence intensity (0.85) corresponding to the outer borders of the IPC, which allows estimation of the dimensions (the "diameter") of the IPC.

Of other means known to be used for the intracellular viscosity measurements (reviewed in Dijksterhuis et al. 2007), a methodology based on the phenomenon of fluorescence polarization was shown to be suitable for the single cell studies (Dix \& Verkman, 1990; Fushimi, Verkman, 1991). The background for the apparent viscosity assessments by the fluorescence polarization is the following (Lakowicz, 2006).

The extent of fluorescence polarization of a fluorophore emission is quantitatively assessed by the anisotropy (r):

$$
\mathbf{r}=\left(\mathbf{I}_{\mathbf{V V}}-\mathbf{G} \times \mathbf{I}_{\mathbf{V H}}\right) /\left(\mathbf{I}_{\mathrm{VV}}+\mathbf{2} \mathbf{G} \times \mathbf{I}_{\mathbf{V H}}\right),
$$

where $\mathbf{I}$ is the fluorescence light intensity; the subscripts $\mathbf{V}$ and $\mathbf{H}$ indicate vertically and horizontally polarized light, respectively; the order of the subscript corresponds to the excitation and the emission light, respectively; $\mathbf{G}$ is the factor that corrects for polarization bias in the instrument.

Fluorescence anisotropy of a molecule depends on the viscosity of the medium according to the Perrin equation:

$$
1 / \mathbf{r}=1 / \mathbf{r}_{0}+\tau R T / r_{0} \eta V,
$$

where $\mathbf{r}$ is the fluorescence anisotropy, $\mathbf{r}_{\mathbf{0}}$ is the fundamental anisotropy (constant), $\boldsymbol{\tau}$ is the fluorescence lifetime, $\mathbf{R}$ is the gas constant, $\mathbf{T}$ is the thermodynamic temperature, $\boldsymbol{\eta}$ is the viscosity, $\mathbf{V}$ is the volume of the rotating molecule.

For the intracellular apparent viscosity assessment, measurements of the fluorescence anisotropy should be done with a fluorescent probe which must be located in a compartment of interest and does not bind to other molecules or structures (Dix, Verkman, 1990). In case of the yeast vacuoles, the most promising candidate for the fluorescent probe could be quinacrine. This fluorescent dye was shown to be concentrated in the $S$. cerevisiae vacuoles by the $\mathrm{pH}$ gradient generated by the vacuolar V-ATPase. Also, upon the $\mathrm{pH}$ gradient discharge by ammonium acetate, it rapidly flowed out of the vacuoles without any noticeable binding (Weisman et al. 1987).

The aim of this part of the study was to evaluate the apparent viscosity within the vacuoles of the single $S$. cerevisiae cells by steady-state fluorescence anisotropy measurements of quinacrine using wide-field fluorescence 
polarization microscopy (Lakowicz 2006) combined with computer image analysis.

Staining pattern of the intact yeast cells by quinacrine is shown in Fig. 10. It clearly indicated the effective intracellular quinacrine accumulation from the medium with significantly higher levels in the vacuoles. Upon ATP depletion of the cells, either prior or after quinacrine accumulation, there was no detectable quinacrine within vacuoles (not shown). These data indicated that, as it was reported by others (Weisman et al. 1987), quinacrine could be accumulated specifically inside the vacuoles of the $S$. cerevisiae cells. This accumulation was due to the $\mathrm{pH}$ gradient generated by the vacuolar V-ATPase, as it was effectively reversed by the ATP depletion by treatment with $5 \mathrm{mM}$ 2-deoxy-D-glucose $+5 \mathrm{mM}$ sodium azide. Importantly, there was no any detectable quinacrine in the vacuoles upon ATP depletion that was a strong evidence of no binding of the dye inside the vacuoles. To test, whether quinacrine can be used as a viscosity probe, its fluorescence anisotropy was measured by conventional fluorimetry in the solutions of sucrose of various viscosities. The quinacrine anisotropy obeyed the Perrin equation (3) (data not shown). It means that the quinacrine fluorescence anisotropy could be used to measure the apparent viscosity of the vacuolar milieu.

Quinacrine fluorescence intensity was assessed in a cell vacuole in corresponding images selected as the region of interest (ROI "Oval Selection") using "Analyze"plugin ("ROI Manager" option) as the "Mean Gray Value". The obtained data were in the arbitrary units of the 8-bit gray scale $(0-256$ units). Three to five images of the same field for a given combination of the polarizer's positions were analyzed, and the mean values of the fluorescence intensities were used for the anisotropy calculations according to the formulas (2) and (3). G factor of the microscope setup has been evaluated according to the formulae (2) using the images of the fluorescing vacuoles. It was found to be 1.1.

The fluorescence anisotropy of quinacrine was measured in the vacuoles of 39 cells. From cell to cell, this parameter varied in a range from 0.032 up to 0.086 . Taking into account that there was no noticeable quinacrine binding inside the vacuoles and using the Perrin plot of quinacine in sucrose solutions as a calibration curve, apparent viscosity of the vacuolar milieu was calculated for each cell. High heterogeneity of the studied cell population with regard to the apparent viscosity within vacuoles in a range from $3.5 \pm$ $0.4 \mathrm{cP}$ to $14.06 \pm 0.64 \mathrm{cP}$ has been found. It is worth noting that the vacuolar viscosity values for four $S$. cerevisiae cells assessed by the IPC Brownian motion (see above) appeared to be within this range. No any reasonable relation between the size of the vacuoles and their lumen viscosity was revealed. On the other hand, there was a characteristic distribution of the frequencies of the cells with the viscosities within certain limits. In the studied population, the cells with the apparent viscosity values in the range from 5 to $6 \mathrm{cP}$ were the most frequent (16 cells of the total 39) that corresponded to approx. $30 \%$ sucrose solution.
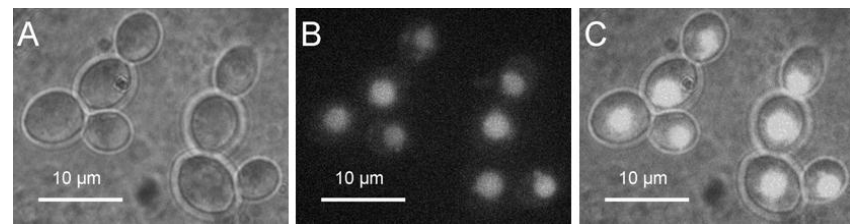

Figure 10. Vacuoles in the $S$. cerevisiae cells stained by quinacrine. A transmission mode; $\mathrm{B}$ - fluorescence mode; $\mathrm{C}$ - superimposed $\mathrm{A}$ and $\mathrm{B}$.

\section{Conclusions}

In this study three quantitative processing algorithms of the ImageJ software were tailored for microfluorimetric studies at subcellular level (as far as resolution of the widefield fluorescence microscopy enabled) on relatively small eukaryotic single cells of the live (not fixed) yeast $S$. cerevisiae. The main results obtained can be summarized as follows.

1. Using an approach called 'pseudospectral analysis', intracellular distribution of anthracycline anticancer drug doxorubicin (DR) along with fluorescent DNA markers DAPI and ethidium (E) were investigated. It was established that all three dyes were located in the nuclei and in the mitochondria. In contrast to DAPI, which interacted only with DNA, an appreciable fraction of DR and $\mathrm{E}$ was probably bond to the mitochondrial membranes. Upon combined addition, DAPI competed with DR and E for binding sites on DNA. This approach may find its application in designing new DNA-targeted drugs at the stage of preliminary assessment of their interaction with eukaryotic cells with the yeast S.cerevisiae as a model.

2. Movement of insoluble polyphosphate complexes (IPCs) stained by DAPI in the vacuoles of the cells was photorecorded and shown to be Brownian motion. On fluorescein isothyocyanate-labelled fluorescing latex microspheres, a methodology was developed for measuring a fluorescing particle's two-dimensional (2D) displacements and its size. Using this methodology, in four yeast cells, the 2D displacements and sizes of the IPCs were evaluated. Apparent viscosity values in the vacuoles of the cells, computed by the Einstein-Smoluchowski equation using the obtained data, were found to be $2.16 \pm 0.60,2.52 \pm 0.63$, $3.32 \pm 0.9$ and $11.3 \pm 1.7 \mathrm{cP}$.

3. Fluorescent dye quinacrine was shown to be specifically accumulated within the vacuoles of the cells. There was no detectable binding of the dye within the vacuoles. The fluorescence anisotropy of quinacrine was measured in the vacuoles of 39 cells. From cell to cell, this parameter changed in the range $0.032-0.086$. Using the Perrin plot as a calibration curve, apparent viscosity values of the vacuolar milieu were calculated for each cell. The population of the cells studied was heterogeneous with regard to vacuolar viscosity, which was in the range $3.5 \pm 0.4$ - $14.06 \pm 0.64 \mathrm{cP}$. There was a characteristic distribution of the frequencies of cells with apparent viscosities within certain limits, and cells with viscosity values in the range 5 $6 \mathrm{cP}$ were the most frequent.

\section{References}


[1] L. Angeloni, G. Smulevich, and M.P. Marzocchi, "Absorption, fluorescence and resonance Raman spectra of adriamycin and its complex with DNA." Spectrochim. Acta, Part A: Mol. Spectrosc. vol. 38, pp. 213-217, 1982.

[2] H. C. Berg, Random walks in biology. Princeton: Princeton University Press, 1983. pp. 11-72.

[3] D. Botstein, S. A. Chervitz, J. M. Cherry, "Yeast as a model organism." Science, vol. 277, pp. 1259-1260, 1997.

[4] S. Bolte, and F. Cordelieres, "A guided tour into subcellular colocalization analysis in light microscopy." J. Microscopy, vol. 224, pp. 213-232, 2006.

[5] C. A. Combs, "Fluorescence microscopy: a concise guide to current imaging methods". Curr. Protoc. Neurosci. Chapter 2, Unit 2.1, Jan 2010 .

[6] J. Dijksterhuis, J. Nijsse, F. A. Hoekstra, E. A. Golovina, "High Viscosity and Anisotropy Characterize the Cytoplasm of Fungal Dormant Stress-Resistant Spores." Eukaryotic Cell, vol. 6, pp. 157170, 2007.

[7] J. A. Dix, and A. S. Verkman, "Mapping of fluorescence anisotropy in living cells by ratio imaging. Application to cytoplasmic viscosity". Biophys. J. vol. 57 , pp. 231-240, 1990.

[8] K. W. Dunn, M. M. Kamocka, and J. H. McDonald, "A practical guide to evaluating colocalization in biological microscopy". Am. J. Physiol. Cell Physiol. vol. 300, pp. C723-C742, 2011.

[9] M. J. Egorin, R. E. Clawson, J. L. Cohen, L. A. Ross, and N. R.Bachur, "Cytofluorescence Localization of Anthracycline Antibiotics." Cancer Research, vol. 40, pp. 4669-4676, 1980.

[10] K. Fushimi, and A. S. Verkman, "Low Viscosity in the Aqueous Domain of Cell Cytoplasm Measured by Picosecond Polarization Microfluofimetry." J. Cell Biol. vol. 112 , pp. 719-725, 1991.

[11] L. Gallois, M. Fiallo, A. Laigle, W. Priebe, and A. Garnier-Suillerot, "The overall partitioning of anthracyclines into phosphatidylcontaining model membranes depends neither on the drug charge nor the presence of anionic phospholipids." Eur. J. Biochem. vol. 241, pp. 879-887, 1996.

[12] M. Giglia, S. M. Dogliac, J. M. Millota, L. Valentinib, and M. Manfaita, "Quantitative Study of Doxorubicin in Living Cell Nuclei by Microspectrofluorometry." Biochimica et Biophysica Acta, vol. 950, pp. 13-20, 1988.

[13] R. P. Haugland, Handbook of Fluorescent Probes and Research Chemicals, 6th Ed., Molecular Probes: Origon, 1996, pp. 144-152.

[14] J. R. Lakowicz, Principles of of fluorescence spectroscopy. 3rd Ed., Springer Science + Business Media, LLC, 2006, pp. 353-383.

[15] J.-B. Le Pecq, C.and Paoletti, "A Fluorescent Complex Between Ethidium Bromide and Nucleic Acids: Physical-Chemical Characterization." Journal of Molecular Biology, vol. 14, pp. 87-106, 1967.

[16] W. H. Mager, and J. Winderickx, "Yeast as a model for medical and medicinal research." Trends Pharmacol. Sci., vol. 26, pp. 265-273, 2005.

[17] Yeast as tool in cancer research. J.L. Nitiss, and J.Heitman, Eds. Springer, Dotdrecht, 2007.

[18] O. Ronneberger, D. Baddeley, F. Scheipl, P. J. Verveer, H. Burkhardt, C. Cremer, L. Fahrmeir, T. Cremer, and B. Joffe, "Spatial quantitative analysis of fluorescently labeled nuclear structures: problems, methods, pitfalls." Chromosome Res. vol. 16, pp. 523-562, 2008.

[19] V. Saks, N. Beraud, and T. Wallimann, "Metabolic Compartmentation - A system level property of muscle cells". Int. J. Mol. Sci. vol. 9, pp. 751-767, 2008.

[20] M. L. Sheely, "Glycerol Viscosity Tables." Ind. Eng. Chem. vol. 24, no. 9, pp. 1060-1064, 1932.

[21] T. Suzuki, K. Fujikura, T. Higashiyama, and K.Takata, "DNA Staining for Fluorescence and Laser Confocal Microscopy." The Journal of Histochemistry \& Cytochemistry, vol. 45, pp. 49-53, 1997.

[22] D. Tsygankov, P. H. Chu, H. Chen, T. C. Elston, and K. M. Hahn, "User-friendly tools for quantifying the dynamics of cellular morphology and intracellular protein clusters." Methods Cell Biol. vol. 123, pp. 409-427, 2014.

[23] J.C. Waters, "Accuracy and precision in quantitative fluorescence microscopy". J. Cell Biol. vol. 185, pp. 1135-1148, 2009.

[24] D. H.Williamson, and D. J. Fennell, "Visualization of yeast mitochondrial DNA with the fluorescent stain "DAPI"," Methods Enzymol. vol. 56, pp. 728-733, 1979.
[25] K. Urech, M. Dürr, Th. Boller, A. Wiemken, J. Schwencke, "Localization of polyphosphate in vacuoles of Saccharomyces cerevisiae". Arch Microbiol., vol.116, no. 3, pp. 275-278, 1978.

[26] L.S. Weisman, R. Bacallao, W. Wickner, "Multiple methods of visualizing the yeast vacuole permit evaluation of its morphology and inheritance during the cell cycle." J. Cell Biol. vol. 105, pp. 15391547, 1987.

[27] J.M. Wiame, "Etude d'une substance polyphosphoree, basophile et metachromatique chez les levures". Biochim Biophys Acta, vol. 1, pp. 234-255, 1947.

[28] F. Yanga, S. S. Tevesa, C. J. Kempb, and S. Henikoffa, "Doxorubicin, DNA Torsion, and Chromatin Dynamics." Biochimica et Biophysica Acta, vol. 1845, pp. 84-89, 2014.

About Author:

Evgeny Puchkov, biophysicist, Doctor of Sciences (the highest Scientific Degree in Russia), Leading Researcher and Microbial Preservation Technology Supervisor at All-Russian Collection of Microorganisms, Skryabin Institute of Biochemistry and Physiology of Microorganisms, Russian Academy of Sciences, Pushchino, Russia.

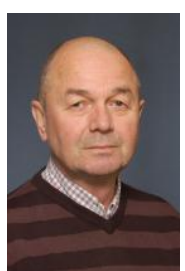

